\title{
Comparison of Guideline Recommendations with Daily Practice in Patients with Renal Cell Carcinoma
}

\author{
B. Schlenker ${ }^{1}$, M. Seitz ${ }^{1}$, M. J. Bader ${ }^{1}$, R. Ganzer ${ }^{2}$, D. Tilki ${ }^{1}$, F. Bayrle ${ }^{1}$, O. Reich ${ }^{1}$, M. Staehler ${ }^{1}$, \\ A. Bachmann ${ }^{3}$, C. G. Stief ${ }^{1}$, C. Gratzke ${ }^{1}$
${ }^{1}$ Department of Urology, University Hospital Grosshadern, Ludwig-Maximilians- University Munich, Germany, ${ }^{2}$ Department of Urology, Krankenhaus St. Josef, University of Regensburg, Germany, ${ }^{3}$ Department of Urology, Basel University Hospital, Switzerland

\begin{abstract}
Objectives and Aims: To compare guideline recommendations with daily practice patterns in a German patient cohort with renal cell carcinoma.

Patients and Methods: 81 patients with T1 oder T2 renal cell carcinoma (RCC) were included in this prospective single-center study. All patients were operated in a single institution either by open radical nephrectomy (ORN) or nephron sparing surgery (NSS). Patients and doctors were evaluated using a written questionnaire with a follow-up of 12 months. Follow-up intervals, follow-up modalities (e. g. imaging modalities, laboratory controls of blood and urine) and the call on psycho-oncological support were evaluated. Results: The majority of patients $(72 \%)$ were followed up by their urologists. Follow-up examinations included abdominal ultrasound, urine and blood diagnostics, conventional chest x-rays, computed tomography (CT) of abdomen, chest or head or abdominal Magnetic Resonance Imaging (MRI). There were no significant differences between patients operated by ORN or NSS. In total, $12.5 \%$ of patients were asking for psycho-oncological support.

Conclusions: In general, patients were followed up according to existing guideline recommendations. Only a small proportion of patients asked for psycho-oncological treatment.
\end{abstract}

Key words: renal cell carcinoma; oncological follow-up; open radical nephrectomy; nephron-sparing surgery

\section{INTRODUCTION}

The incidence of renal cell carcinoma (RCC) is estimated to be 46.000 per year in the European Union with an annual increase of $2-3 \%[1,2]$. One third of patients is dying within 5 years after first diagnosis [3]. Men develop renal cell carcinoma more often with a $1.5: 1$-ratio compared to women. With a mean age of 65 years at first diagnosis, $25-35 \%$ of patients present with metastatic disease [4].

Due to improved imaging techniques such as ultrasound and CT-scanners in the developed countries, tumors are nowadays discovered incidentally during rou- tine scans. These tumors are often asymptomatic, smaller in size and of lower stage $[5,6]$.

According to the European Association of Urology (EAU) guidelines on RCC, surgical options for the primary tumor include radical nephrectomy, organ preserving partial nephrectomy and laparoscopic nephrectomy [7]. For small and/or peripheral located tumors an organ preserving approach is recommended.

The aim of follow-up after surgical therapy of RCC is to monitor and identify post-operative complications, renal function, local recurrence, recurrence in the contralateral kidney and development of metastases [7]. Surprisingly, the EAU guidelines on RCC state that there is no consensus about the ideal oncological follow-up for patients after surgery for RCC; neither do they provide a risk stratification for different follow-up strategies of patients operated by organ preserving techniques or radical surgery.

We therefore conducted a prospective study to investigate how the follow-up of patients with a history of RCC is performed in daily practice. We hereby focused on patients who had undergone open radical nephrectomy (ORN) or open nephron-sparing surgery (NSS) for T1 and T2 RCC. We investigated follow-up modalities and frequency and compared it to the existing guidelines. Furthermore we examined if the follow-up modalities were different when performed by a certified urologist or general practitioner. Finally patient's preference towards psycho-oncological support was assessed.

\section{Patients And Methods}

\section{Patients}

In this prospective single-center study patients undergoing surgery at our institution for renal cell carcinoma stage T1 or T2 were included. All analyses were conducted according to the guidelines of the Local Ethical Committees. Between January and December 200581 patients (29 female, 52 male) with a mean age of $63 \pm 3$ years met the inclusion criteria. 37 patients (45.7\%) underwent ORN and $44(54.3 \%)$ NSS. Pa- 
tients and doctors were evaluated using a written questionnaire with a follow-up of 12 months. The following parameters were examined: which specialist (urologist, nephrologist, oncologist, specialist for internal medicine) was performing oncological follow-up, follow-up intervals, follow-up modalities (e. g. imaging modalities, laboratory controls of blood and urine) and the call on psycho-oncological support.

\section{SURGERY}

Radical nephrectomy was performed as described before $[8,9]$. Briefly, the kidney was accessed according to the principles of Robson through a flank incision, with early control of the renal artery and vein, and complete extraction of Gerota's fascia. Adrenalectomy was used only if preoperative screening or intraoperative palpation revealed suspect masses. Lymphadenectomy was not used regularly, but only if suspect nodes were detected during preoperative staging or surgery. NSS was usually done through a flank incision with a retroperitoneal approach. Clamping was used for complex resections, with smaller peripheral lesion not being resected under ischaemia [10].

\section{RESUlts}

Oncological follow-up was performed by urologists in $72 \%$ of patients, by the general practitioners in $20 \%$, by nephrologists in $9 \%$, by oncologists in $5 \%$ and by specialists for internal medicine in $4 \%$ of cases (see Fig. 1).

Follow-up examinations included abdominal ultrasound, urine and blood diagnostics, conventional chest $\mathrm{x}$-rays, computed tomography (CT) of abdomen, chest or head or abdominal Magnetic Resonance Imaging (MRI). For detailed follow-up modalities see Figure 2.

The follow-up modalities were different for urologists and non-urologists. All technical follow-up modalities were used more often by urologists than by non-urologists. For all differences see Figure 3.

The majority of patients underwent follow-up every 3 months during the first year after surgery. Most patients were willing to actively participate in oncolgical follow-up. See Figure 4 for detailed information about follow-up intervals.

In total, $12.5 \%$ of patients asked for psycho-oncological support (see Figure 5 for details and differences between ORN and NSS).

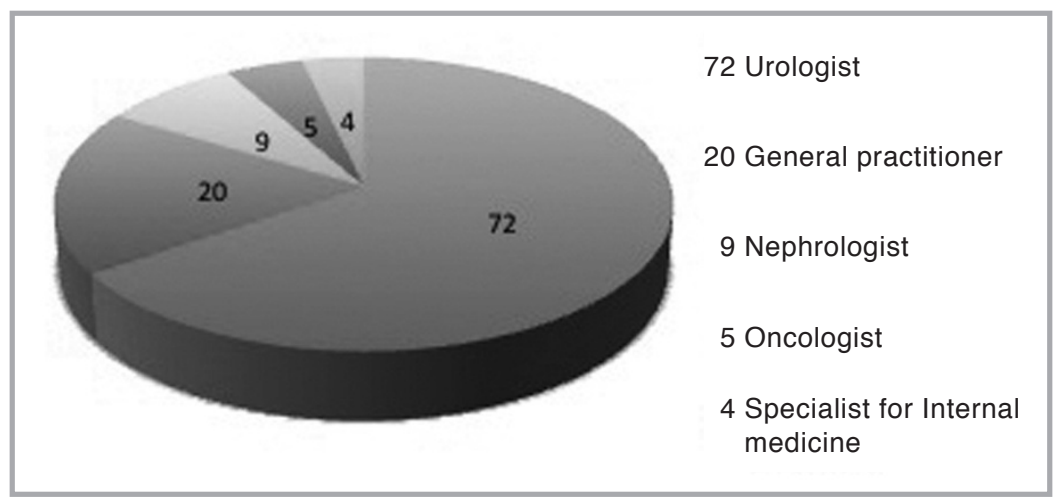

Fig. 1. Medical speciality performing oncological follow-up.

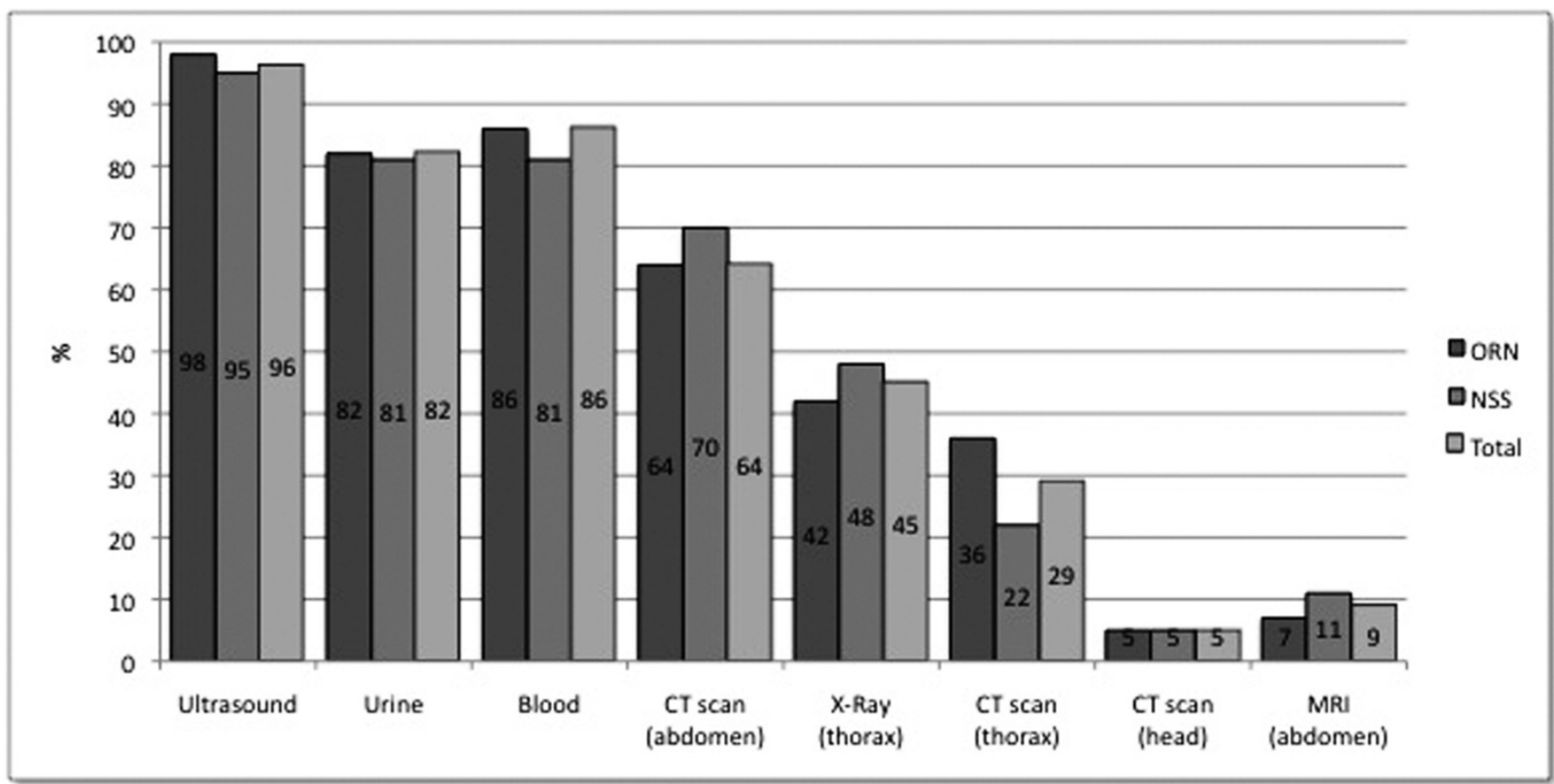

Fig. 2. Follow-up modalities for ORN and NSS. 


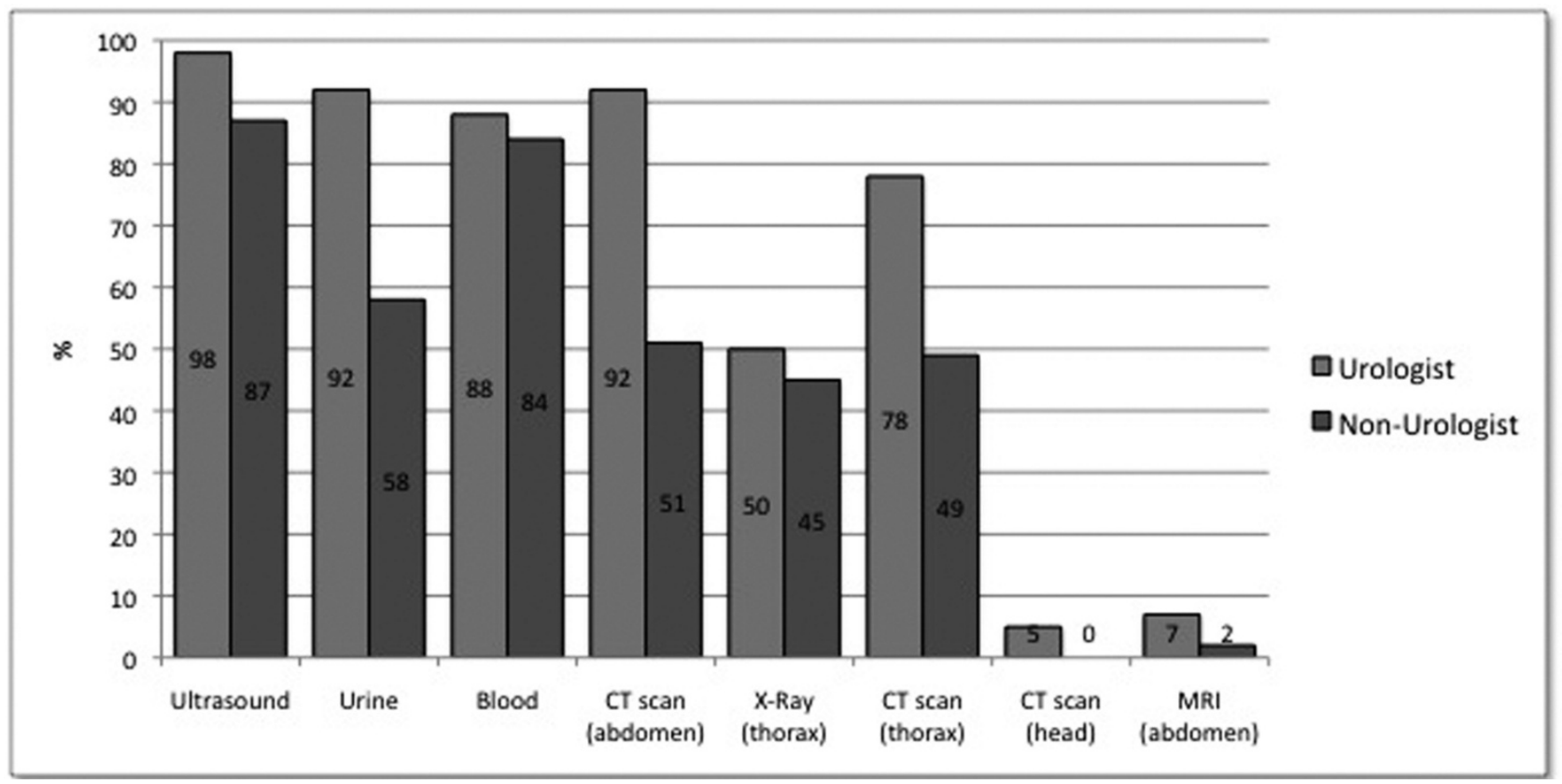

Fig. 3. Differences in follow-up modalities between urologists and non-urologists.

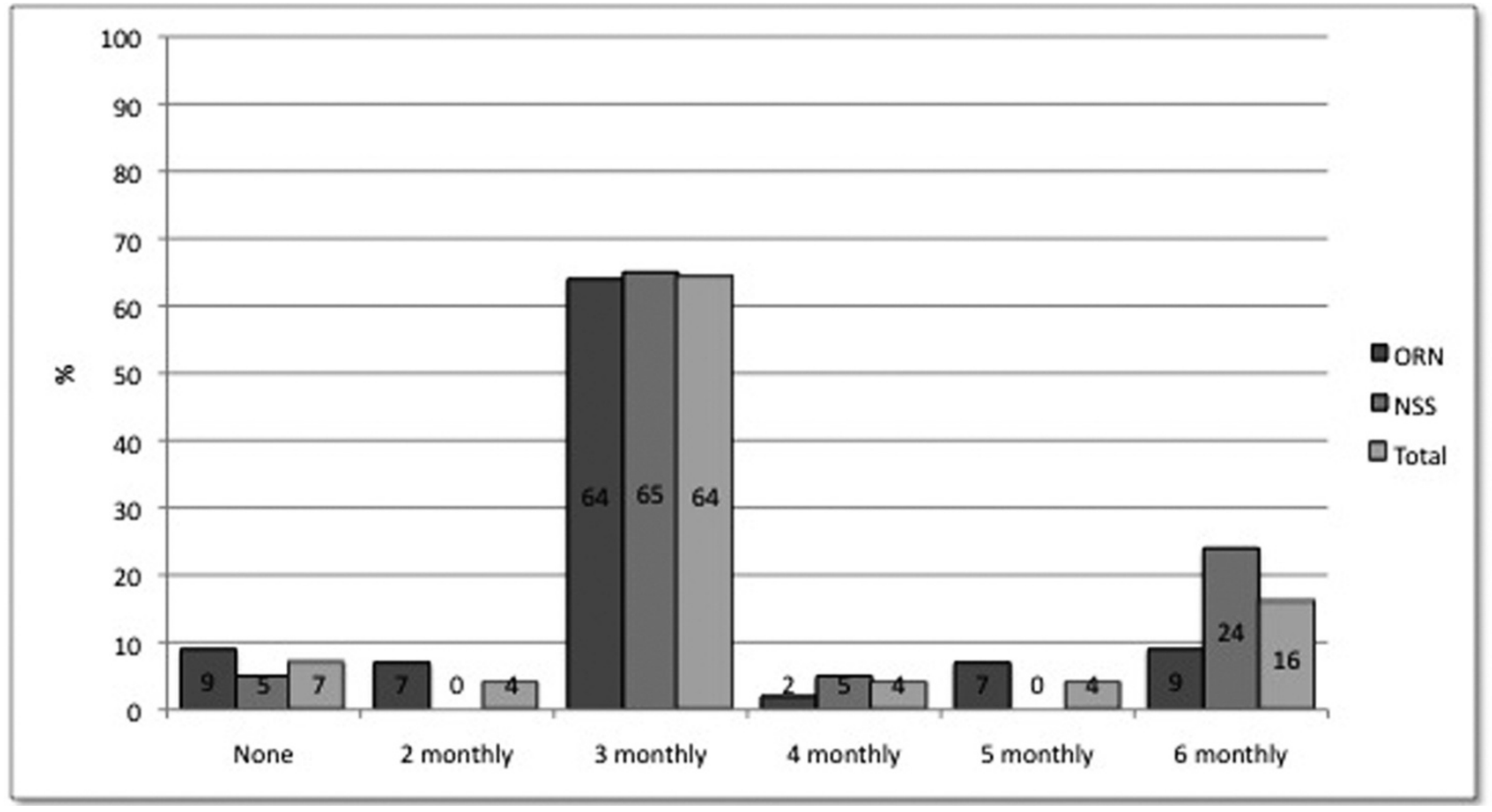

Fig.4. Follow-up intervals after ORN and NSS.

\section{DisCUSSION}

Renal cell carcinoma is a frequent and aggressive tumor entity. Oncological follow-up is of major concern as local and contralateral recurrence occurs $(1.2 \%$ and $1.8 \%$ respectively) [11]. Early detection of recurrence is of utmost importance as cytoreductive surgery is the key for successful treatment [12, 13]. Surprisingly there is no consensus on the ideal postoperative surveillance strategy [7]. To assess the risk of either local or contralateral recurrence several prognostic factors were identified and scoring systems and algorithms were developed [14-17]. Despite the reliable prediction of recurrence in a defined cohort of patients (e. g. patients treated at the University of California at Los Angeles) these nomograms have not gained wide acceptance in Germany [18].

To define the ideal follow-up modality it is essential to know time and localization of recurrence and to adapt the imaging modalities accordingly. Larger autopsy studies have shown that even clinically undetected RCC spreads to lung $(2-14 \%)$, bone $(1-7.6 \%)$ 


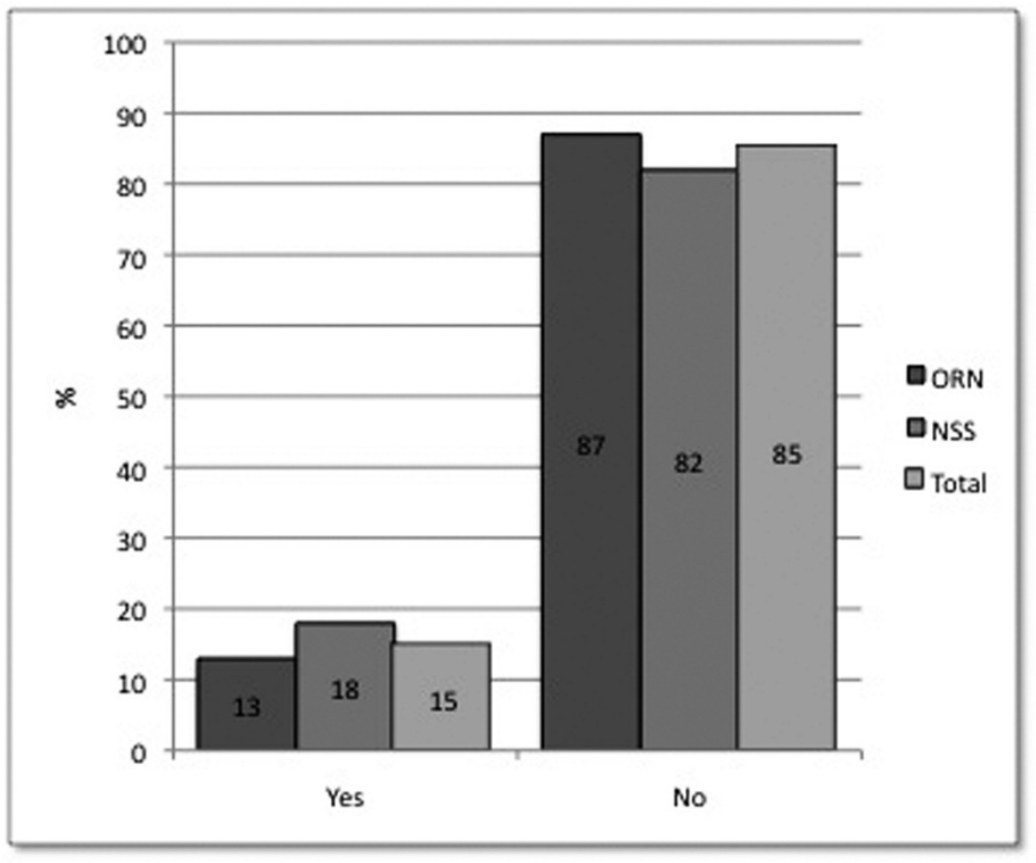

Fig. 5. Patients calling upon psycho-oncological support. and liver $(0-7.4 \%)$ [19-21]. Therefore these locations have to be monitored closely during follow up. For small tumors the risk of recurrence seems to be independent of radical or organ sparing surgery [12, 19, 22, 23]. Therefore the EAU guidelines suggest rather a risk stratification by histopathological factors like grading, pathological stage and lymph node status than the type of surgery performed [7]. In low-risk patients routine $\mathrm{CT}$ scans are not recommended except in cases of possible tumour associated symptoms (weight loss, haematuria, painful bones). Only for intermediate and high-risk patients close follow up by CT scans might be indicated.

Besides the most likely location of recurrent disease, the average time until recurrence is decisive for follow-up recommendations. According to the Mayo Scoring system [16] recurrence in the first year occurs in patients with low-risk, intermediate-risk and highrisk in $0.5,9.6$ and $42.3 \%$, respectively. After 5 years the recurrence rate raises up to 2.9, 26.2 and $68.8 \%$, respectively. As most recurrence occur within 5 years after first diagnosis close follow-up might be omitted after this time [24].

To the best of our knowledge there is no study investigating the follow-up modalities of patients with renal cell carcinoma in Germany. Neither it is known if patients prefer follow-up examinations by their urologist or general practitioner and how closely patients are followed up.

In our cohort, in $72 \%$ of patients were examined by their urologist, followed by general practitioners $(20 \%)$ and nephrologists (9\%). This distribution might be due to the specific health system in Germany in which general practitioners play the central role in referring oncologic patients to specialists (e. g. urologists). Many patients obviously tend to undergo follow-up at the doctor who actually diagnosed the tumor. As ultrasound is broadly available in developed countries a considerable number of tumors are detected during routine check-up examinations by general practitioners.
The types of technical examinations were the same for patients after ORN and NSS. In 96\% ultrasound was used, laboratory controls of urine and blood were used in more than $80 \%$. CT scans of abdomen and chest were used in $64 \%$ respectively $29 \%$ of patients. This is in line with most follow-up recommendations suggesting CT scans every 6 months for 2 years for intermediate- and high-risk patients. CT scans of head $(5 \%)$ or abdominal MRI $(9 \%)$ were limited to selected cases or indications as suggested by the EAU.

Interestingly there were significant differences in the examination modalities employed between urologists and non-urologist health professionals. Urologists applied all of them more often with the largest discrepancy for imaging modalities. This might be an indication that urologist more often stick to guideline recommendations by urological associations.

Nearly two thirds of patients were followed every three months, which seems to be a reasonable interval [7]. On the other hand up to $9 \%$ of patients were not under surveillance at all, indicating the need of a better patient education.

Only a small proportion of patients $(13-18 \%)$ was asking for psycho-oncological support. It is known from larger studies that up to $41 \%$ of patients with tumor disease are in need of professional psycho-oncological support [25]. The discrepancy might be due to a low tumor stage (most of them T1) in our patients contributing to better well being compared to other studies. As many patients might benefit of psycho-oncological support, it should be routinely and actively offered during hospital stay.

\section{CONCLUSIONS}

Patients in our cohort appeared to be well followedup according to existing guidelines. In this cohort with low tumor stages only the minority of patients asked for psycho-oncological support. 


\section{REFERENCES}

1. Lindblad P. Epidemiology of renal cell carcinoma. Scand J Surg 2004;93:88-96.

2. Mathew A, Devesa SS, Fraumeni JF, Jr., Chow WH. Global increases in kidney cancer incidence, 1973-1992. Eur J Cancer Prev 2002;11:171-8.

3. Lipworth L, Tarone RE, McLaughlin JK. The epidemiology of renal cell carcinoma. J Urol 2006;176:2353-8.

4. Hock LM, Lynch J, Balaji KC. Increasing incidence of all stages of kidney cancer in the last 2 decades in the United States: an analysis of surveillance, epidemiology and end results program data. J Urol 2002;167:57-60.

5. Luciani LG, Cestari R, Tallarigo C. Incidental renal cell carcinoma-age and stage characterization and clinical implications: study of 1092 patients (1982-1997). Urology 2000;56:58-62.

6. Patard JJ, Rodriguez A, Rioux-Leclercq N, Guille F, Lobel B. Prognostic significance of the mode of detection in renal tumours. BJU Int 2002;90:358-63.

7. Ljungberg B, Hanbury DC, Kuczyk MA, et al. Renal cell carcinoma guideline. Eur Urol 2007;51:1502-10.

8. Gratzke C, Seitz M, Bayrle F, et al. Quality of life and perioperative outcomes after retroperitoneoscopic radical nephrectomy (RN), open $\mathrm{RN}$ and nephron-sparing surgery in patients with renal cell carcinoma. BJU Int 2009;104:470-5.

9. Robson CJ. Radical nephrectomy for renal cell carcinoma. J Urol 1963;89:37-42.

10. Staehler M, Haseke N, Stadler T, et al. Renal surgery in the elderly: morbidity in patients aged $>75$ years in a contemporary series. BJU Int 2008;102:684-7.

11. Bani-Hani AH, Leibovich BC, Lohse CM, Cheville JC, Zincke $\mathrm{H}$, Blute ML. Associations with contralateral recurrence following nephrectomy for renal cell carcinoma using a cohort of 2,352 patients. J Urol 2005;173:391-4.

12. Itano NB, Blute ML, Spotts B, Zincke H. Outcome of isolated renal cell carcinoma fossa recurrence after nephrectomy. J Urol 2000;164:322-5.

13. Sandhu SS, Symes A, A'Hern R, et al. Surgical excision of isolated renal-bed recurrence after radical nephrectomy for renal cell carcinoma. BJU Int 2005;95:522-5.

14. Kattan MW, Reuter V, Motzer RJ, Katz J, Russo P. A postoperative prognostic nomogram for renal cell carcinoma. J Urol 2001;166:63-7.

15. Lam JS, Shvarts O, Leppert JT, Pantuck AJ, Figlin RA, Belldegrun AS. Postoperative surveillance protocol for patients with localized and locally advanced renal cell carcinoma based on a validated prognostic nomogram and risk group stratification system. J Urol 2005;174:466-72; discussion 72; quiz 801.
16. Leibovich BC, Blute ML, Cheville JC, et al. Prediction of progression after radical nephrectomy for patients with clear cell renal cell carcinoma: a stratification tool for prospective clinical trials. Cancer 2003;97:1663-71.

17. Ljungberg B, Alamdari FI, Rasmuson T, Roos G. Followup guidelines for nonmetastatic renal cell carcinoma based on the occurrence of metastases after radical nephrectomy. BJU Int 1999;84:405-11.

18. Zisman A, Pantuck AJ, Wieder J, et al. Risk group assessment and clinical outcome algorithm to predict the natural history of patients with surgically resected renal cell carcinoma. J Clin Oncol 2002;20:4559-66.

19. Hafez KS, Novick AC, Campbell SC. Patterns of tumor recurrence and guidelines for followup after nephron sparing surgery for sporadic renal cell carcinoma. J Urol 1997;157:2067-70.

20. Johnsen JA, Hellsten S. Lymphatogenous spread of renal cell carcinoma: an autopsy study. J Urol 1997;157:450-3.

21. Levy DA, Slaton JW, Swanson DA, Dinney CP. Stage specific guidelines for surveillance after radical nephrectomy for local renal cell carcinoma. J Urol 1998;159:11637.

22. Schrodter S, Hakenberg OW, Manseck A, Leike S, Wirth MP. Outcome of surgical treatment of isolated local recurrence after radical nephrectomy for renal cell carcinoma. J Urol 2002;167:1630-3.

23. Patard JJ, Shvarts O, Lam JS, et al. Safety and efficacy of partial nephrectomy for all T1 tumors based on an international multicenter experience. J Urol 2004;171:2181-5, quiz 435.

24. Ebert T, Fischer C. [When is aftercare useful in renal cell cancer?]. Urologe A 2005;44:1018, 20-3.

25. Goerling U, Odebrecht S, Schiller G, Schlag PM. [Need for psychosocial care in in-patients with tumour disease. Investigations conducted in a clinic specializing in tumour surgery]. Chirurg 2006;77:41-6; discussion 6.

Received: October 25, 2009 / Accepted: November 10, 2009

\section{Address for correspondence:}

Boris Schlenker, M.D.

Department of Urology

University Hospital Grosshadern

Ludwig-Maximilians-University Munich

Marchioninistr. 15

81377 Munich

Germany

Tel.: +49-89-7095-0

Fax: +49-89-7095-8890

E-mail: boris.schlenker@med.uni-muenchen.de 